\title{
Examining Developmental Changes in Children's Motor Imagery: A Longitudinal Study
}

\author{
Steffie Spruijt ${ }^{1}$, Marijtje L. A. Jongsma ${ }^{1}$, John van der Kamp ${ }^{2,3}$, and Bert Steenbergen ${ }^{1,4}$
}

\author{
1 Radboud University, Behavioural Science Institute, Nijmegen, the Netherlands \\ ${ }^{2}$ Vrije Universiteit Amsterdam, Research Institute Move, Amsterdam, the Netherlands \\ ${ }^{3}$ University of Hong Kong, Institute of Human Performance, Hong Kong, China \\ ${ }^{4}$ Australian Catholic University, School of Psychology, Melbourne, Australia
}

ABSTRACT

Using a longitudinal design, the present study examined developmental changes in the employment of (motor) imagery strategies on the hand laterality judgment (HLJ) task in children. All children $(N=23)$ participated three times, at ages of 5, 6, and 7 years. Error percentages and response durations were compared to a priori defined sinusoid models, representing different strategies to judge hand laterality. Response durations of correct and incorrect trials were included. Observed data showed that task performance was affected by motor constraints, both in children who performed accurately at 5 years of age and in the children who did not. This is the first study to show that 5-year-olds-even when not successful at the task-employ motor imagery when engaged in this task. Importantly, although the children became faster and more accurate with age, no developmental changes in the employed motor imagery strategy were observed at ages of 5,6, and 7 years. We found that 5 -year-old children are able to use a motor imagery strategy to perform the HLJ task. Although performance on this task improved with age, our analyses showed that the

employed strategy to solve this task remained invariant across age.

motor imagery,

development,

hand laterality judgment,

children

\section{INTRODUCTION}

Motor imagery, or imagining performing a movement without any overt motor behaviour, shares overlapping motor processes with the actual execution of movements. As motor imagery comprises the internal activation of a movement representation from a first-person perspective, it shares many aspects with the actual execution of movements (Decety \& Grezes, 1999; Jeannerod, 1995). In the current study, we examined developmental changes in children's employment of (motor) imagery strategies by testing them once a year for three consecutive years. Thus far, motor imagery development has solely been studied by examining interindividual age differences employing a cross-sectional approach (e.g., Butson, Hyde, Steenbergen, \& Williams, 2014; Caeyenberghs, Tsoupas, Wilson, \& Smits-Engelsman, 2009; Smits-Engelsman \& Wilson, 2012; Spruijt, Jongsma, van der Kamp,
\& Steenbergen, 2015). As we have previously stated, motor imagery development has not yet been studied using a longitudinal design that can reveal intraindividual changes over time (Spruijt, van der Kamp, \& Steenbergen, 2015). Such a design is, however, a critical initial step in capturing the dynamic processes of developmental change in motor ability and cognition (Grammer, Coffman, Ornstein, \& Morrison, 2013; Thelen \& Smith, 1994; Wohlwill, 1970).

A commonly used paradigm to study motor imagery ability is the hand laterality judgment (HLJ) task. Within the HLJ task, participants

Corresponding author: Marijtje Jongsma Ph.D., Radboud University Nijmegen, P.O. Box 9104, 6500 HE Nijmegen, the Netherlands.

E-mail:m.jongsma@donders.ru.nl 
judge whether a picture of a hand displays a left or right hand. The combination of manipulating the angle of rotation (i.e., the degree to which the hand picture is rotated away from the upright position) and the direction of rotation (i.e., away or towards the midline of the body) defines the orientation of the hand stimulus. The angle of rotation can vary from $0^{\circ}$ with the fingers pointing up to $180^{\circ}$ with the fingers pointing down; the direction of rotation can vary between $90^{\circ}$ with the fingers towards the midline of the body (medial orientation) and $270^{\circ}$ with the fingers away from the body (lateral orientation, see Figure 1). Other tasks and methods have been employed as well, for example, mental chronometry tasks (Spruijt et al., 2013). In addition, neuroimaging research on both the HLJ task and mental chronometry tasks has shed light on the involved neural substrates (for a comprehensive review see Hétu et al., 2013). In addition, with respect to the HLJ task, we found that particularly mu-desynchronisation (Ter Horst, van Lier, \& Steenbergen, 2013), and the error-related negativity (Jongsma et al., 2013; Ter Horst, Jongsma, Janssen, van Lier, \& Steenbergen, 2012) were indicative of the use of motor imagery.

Participants can employ different (imagery) strategies to perform the HLJ task. For instance, participants can use motor imagery, in which they judge hand laterality by mentally rotating their own hand into the position of the displayed hand. As the imagined hand rotation involves a motor representation of hand movements, the same motor constraints that impact upon actual hand movements also affect the motor imagery performance (Lust, Geuze, Wijers, \& Wilson, 2006; Parsons, 1987). Furthermore, participants can mentally rotate the displayed hand like any other detached object to perform the HLJ task. This mental imagery strategy is not related to the motor system (i.e., nonmotor imagery) and task performance is therefore not affected by motor constraints (Steenbergen, van Nimwegen, \& Crajé, 2007; Wilson et al., 2004). Alternatively, the HLJ task can be performed without systematically adopting a mental imagery strategy. Instead, participants rely on an abstract rule or visual cue to judge the laterality of the presented hands (as suggested by Ter Horst, van Lier, \& Steenbergen, 2010). For example, for back view stimuli, if the thumb and index finger can be shaped as a capital $L$, it is a left hand.

We have recently introduced a priori defined sinusoid models to determine what strategy participants employ to perform the HLJ task (Spruijt, Jongsma, et al., 2015). See also Table 1 for an overview. The first model predicts that task performance is not systematically affected by manipulations of rotation angle and/or rotation direction, which is the case when participants perform the HLJ task by means of an abstract rule. The second model predicts changes in task performance as a function of rotation angle only. When participants mentally rotate the displayed hand, the task performance decreases when the rotation angle increases (Shepard \& Metzler, 1971). This effect is independent of whether the hand is rotated in the medial or lateral direction. The third model predicts that the direction of rotation affects task performance, suggesting that biomechanically "awkward" hand orientations (i.e., lateral orientations) result in diminished task performance compared to more "comfortable" hand orientations (i.e., medial orientations). This third model represents a motor imagery strategy, as the HLJ task performance is subject to the same motor constraints as actual movements (Parsons, 1987). Taken together, a comparison of the observed data with model-based predicted data enables us to disentangle the combined effects of manipulating rotation angle and direction of rotation on task performance (see Table 1). Consequently, this allows us to determine what strategy is employed by the participants (see also Spruijt, Jongsma, et al., 2015).

With respect to changes in children's motor imagery as a function of age, previous cross-sectional studies using the HLJ task have shown equivocal results in children between 5 and 11 years of age. Krüger and Krist (2009) and Toussaint, Tahej, Thibaut, Possamai, and Badets (2013) argued that the HLJ task performance was more constrained by motor characteristics for 7- versus 5 -year-olds (Krüger \& Krist, 2009) and for 8- versus 6-year-olds (Toussaint et al., 2013). These observations indicate increased motor imagery ability from 5 to 8 years of age. Butson et al. (2014) also found age-related differences in motor imagery ability. However, their results do not suggest a consistent increase in motor imagery across age, as the results of the 8-, 9-, and 11-year-olds did indicate the use of motor imagery, whereas the results of the 7-and 10 -year-olds did not. Moreover, in a recent cross-sectional study, we did not observe any age-related differences in the use of motor imagery on the HLJ task between 5 and 8 years of age (Spruijt, Jongsma, et al., 2015). A common facet of existing studies is the exclusion of erroneous responses and/or individual participants who do not perform the task sufficiently accurately (i.e., do perform at chance level). This is especially evident in young children. As an illustration, Krüger and Krist excluded $40 \%$ of the 5 -year-olds, and Butson et al. even excluded all children of 5 and 6 years, as $73 \%$ of these children did not identify hand laterality above $50 \%$ accuracy. The ability of children to accurately judge hand laterality increases with age; for example, at 7 years of age, only $17 \%$ of the children were not able to perform the task above chance levels in the study of Krüger and Krist. Importantly, however, with the exclusion of inaccurately performing children and erroneous trials, it is likely that insights in the early development of (motor) imagery strategies are biased or overlooked. Specifically, the transition from not performing the task above chance to performing the task above chance may indicate developmental changes in motor imagery that are potentially overlooked if only participants that perform the task accurately are included. So far, it remains unclear whether young children perform the task inaccurately due to an inability to employ motor imagery or inaccuracy while employing motor imagery, or more generally to

\section{TABLE 1.}

Predictive Models to Determine the Employed Strategy

\begin{tabular}{ccccc}
\hline Model & $\begin{array}{c}\text { Rotation } \\
\text { effect }\end{array}$ & $\begin{array}{c}\text { Direction } \\
\text { effect }\end{array}$ & Sinusoid model & Strategy \\
\hline $\begin{array}{c}\text { H0 } \\
\text { model }\end{array}$ & No & No & $\begin{array}{c}\text { Amplitude } \\
=0\end{array}$ & $\begin{array}{c}\text { No mental } \\
\text { imagery }\end{array}$ \\
H1 & Yes & No & $\begin{array}{c}\text { Phase shift } \\
=90^{\circ}\end{array}$ & $\begin{array}{c}\text { Nonmotor } \\
\text { imagery } \\
\text { model }\end{array}$ \\
$\begin{array}{c}\text { H2 } \\
\text { model }\end{array}$ & Possible & Yes & $\begin{array}{c}\text { Phase shift } \\
>90^{\circ} \text { and } \leq 180^{\circ}\end{array}$ & $\begin{array}{c}\text { Motor } \\
\text { imagery }\end{array}$ \\
\hline
\end{tabular}


an inability to understand the task instructions or other limitations in cognitive ability. Therefore, in addition to examining developmental changes in the strategies employed by the children performing above chance, we also analysed imagery strategies of children that did not perform above chance.

Following the approach of our previous cross-sectional study (Spruijt, Jongsma, et al., 2015), we determined the employed strategies by examining the effects of task manipulations on response accuracy and response duration patterns. An important difference to previous work is that, in the current study, we employed a longitudinal design, without excluding data from erroneous responses. With respect to erroneous responses, we predicted that similar effects of rotation angle and/or rotation direction as commonly described for the response durations can be observed. Hence, we hypothesized that young children are already able to engage in motor imagery. More specifically, we expected that younger children are more likely to rely on a motor imagery strategy to solve the task than alternative strategies, but that other processes-such as the ability to correctly distinguish between the abstract concepts of left and right and/or the ability to clearly understand the task instruction-may have obscured this in previous studies. For instance, when children cannot engage in motor imagery, they will perform misguided or guess blindly, instead of attempting to make genuine judgments of hand laterality. If this is the case, then the amount of erroneous responses would not be systematically affected by stimulus manipulations (H0). Alternatively, it might be that children who do not perform above chance level employ a mental rotation strategy. If so, they were expected to produce more erroneous responses on stimuli with larger rotation angles compared to smaller rotation angles (effect of angle of rotation; H1). In line with this assumption, if stimuli with lateral orientations result in more erroneous responses than medial orientations (direction effect; $\mathrm{H} 2$ ), this would suggest a motor imagery strategy.

In the present study, we aimed to determine early developmental changes in the employment of (motor) imagery strategies on the HLJ task, for children performing the task at chance and above chance at 5 years of age. We included children that were 5 years old and followed them longitudinally for three consecutive years to determine whether and how the involvement of imagery strategies on the HLJ task changes at 5, 6, and 7 years of age. This age range was shown to be critical with respect to age-related differences in the ability to accurately perform the HLJ task and age-related differences in motor imagery (e.g., Butson et al., 2014; Caeyenberghs, Tsoupas, et al., 2009; Smits-Engelsman \& Wilson, 2012), which can be related to the maturation of motor processes that are involved in motor imagery during childhood (Caeyenberghs, Wilson, van Roon, Swinnen, \& SmitsEngelsman, 2009). In line with our previous cross-sectional results (Spruijt, Jongsma, et al., 2015), we expected that the response durations of children performing the HLJ task above chance are affected by motor constraints (direction effect; H2) between 5 and 7 years of age. In this group, we did not expect developmental changes in the use of the motor imagery strategy. As we are the first to address motor imagery ability in children who do not perform the HLJ task above chance, we explored whether children who are inaccurate at the task at 5 years of age are engaged in motor imagery, via the examination of response accuracy patterns. Furthermore, we explored whether developmental changes in the employed strategies underlie anticipated improvements in overall HLJ task accuracy between 5 and 7 years of age (see Butson et al., 2014).

\section{METHODS}

\section{Participants}

A total of 23 typically developing, right-handed children participated in the study ( 11 males). The participants were 5 years of age at the moment of the first measurement (mean age at first, second, and third measurement was, respectively, 5.6, 6.6, and 7.6 years; $S D=0.249$ ). Children were recruited from primary schools in the Netherlands and only one drop-out was reported. Children were tested at a quiet office of their school. None of the children had a formal diagnosis of developmental coordination disorder (DCD) or attention deficit hyperactivity disorder (ADHD), as was confirmed by their teacher. Handedness was assessed with a test based on the Edinburgh Handedness Inventory (Oldfield, 1971) that was specifically adapted for 5-7 year old children (Geuze, Lust, \& Bouma, 2009). Children were tested once a year (between mid-March and mid-April) for three consecutive years. Parents provided written informed consent prior to the experiment. The study was approved by the local ethics committee (ECG2012-2402-018). The response duration data of the correct trials from the first measurement was already used in our previous cross-sectional study (Spruijt, Jongsma, et al., 2015).

\section{Material and Procedure}

The experimental procedure is similar to the procedure described in our previous study (for more details, see Spruijt, Jongsma, et al., 2015). Children had to judge whether a picture, which was presented on a computer screen, displayed a left or a right hand. After a white fixation cross was presented, a picture of a hand was shown in the middle of the screen. The child was instructed to press a button with the left hand for a picture of the left hand, and with the right hand for a picture of the right hand. The children were instructed to respond as fast as possible. The picture disappeared after the response was given and the fixation cross was shown until the next stimulus presentation. The children were not allowed to make any hand and/or head rotations during the laterality judgment and the hands were covered with a cloth to prevent a direct visual comparison.

The stimuli were pictures of left and right hands, showing the palm of the hand. The stimuli were presented in six different rotation angles: $0^{\circ}, 60^{\circ}, 120^{\circ}, 180^{\circ}, 240^{\circ}$, and $300^{\circ}$ (see Figure 1). Each hand stimulus was presented three times, resulting in 36 randomly ordered trials. Six practice trials were performed before the start of the experiment. The study employed a three-year longitudinal design during which the participants were measured annually. 
Figure 1. Examples of hand stimuli. Hand stimuli consisted of left and right hands in the palm view, varying in rotation angle and direction of rotation.

\section{Data Analysis}

A binomial distribution ( $p=.50$ for each trial) was used to establish whether or not the children performed the HLJ task above chance level. Individual performance was significantly above chance level when more than 23 out of 36 stimuli were correctly identified. Based on the task accuracy at 5 years of age, we divided the children in two groups; a group of children that was not able to perform the HLJ task above chance level at 5 years of age (Group A) and a group of children that was able to perform the HLJ task above chance level at 5 years of age (Group B).

For the response durations, outlier trials (response duration less than $250 \mathrm{~ms}$ or response duration greater than the mean response duration $+3 \times S D)$ were excluded from further analyses $(1.93 \%$ of the trials). Percentages of erroneous responses and response durations for left and right hand stimuli were pooled and averaged across the repetitions of each of the six rotation angles. To determine the strategy that participants of Groups A and B employed at each age, the averaged observed data were compared to the hypothetical models (see Spruijt, Jongsma, et al., 2015). Error and response duration data were analyzed separately. Goodness of fit $F$-tests were used to model the distribution of the data as a function of the rotation angle and the direction of rotation using GraphPad Prism version 6.07 for Windows (GraphPad Software). It was tested whether the amplitudes differed from 0 ( $\mathrm{H} 0$; nonimagery strategy) at each age. If $\mathrm{H} 0$ was rejected, it was tested whether the phase shift was different from $90^{\circ}$ or not ( $\mathrm{H} 1$; phase shift $=90^{\circ}$, a nonmotor imagery strategy was employed) and whether the phase shift was different from $180^{\circ}$ or not $\left(\mathrm{H} 2\right.$; phase shift $>90^{\circ}$ and $\leq 180^{\circ}$, a motor imagery strategy was employed). A Bonferroni correction was used that resulted in an alpha level of $p=.017$ (three measurements).

To examine whether imagery strategies changed across age, $F$-tests for goodness of fit (GraphPad Prism 6.07) were used to determine whether the error and response duration data at 5, 6, and 7 years of

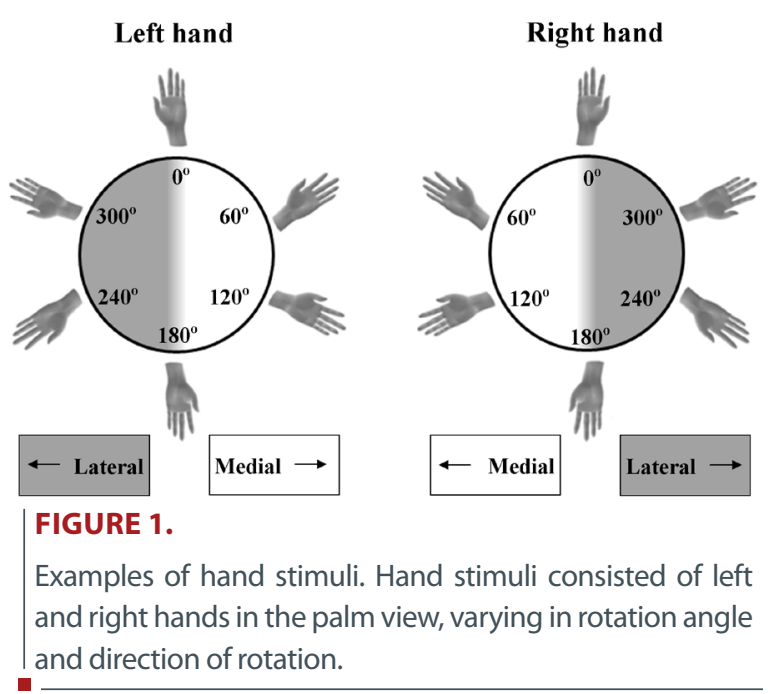

age could be described by the same parameters. This analysis was performed for Groups A and B separately.

\section{RESULTS}

\section{Response Accuracy (Error Percentage)}

Fifteen ( 8 male) out of 23 children did not perform the HLJ task above chance at 5 years of age. These children were assigned to Group A $\left(M_{\text {age }}\right.$ $=5.51 ; S D=0.248$ ). Only three of these children were not able to accurately perform the HLJ task at age 6 and one of them still did not perform above chance at 7 years of age. Group B $\left(M_{\text {age }}=5.76 ; S D=0.171\right)$ consisted of 8 children ( 3 male) that were already able to perform the HLJ task above chance at 5 years of age. One of them did not perform above chance at 6 and 7 years of age.

We described the variation in percentage of erroneous responses as a function of rotation angle and direction in Groups A and B by means of sinusoid curves and tested them to the three a priori defined sinusoid models that reflect the different strategies to perform the HLJ task. The resulting parameters of the sinusoid curves are displayed in Table 2, and the curves are presented in Figure 2. For example, the error data for Group A at 5 years of age could best be described by percentage error $=16.5 \times \sin ($ angle -198$)+51.3$.

The amplitudes of the sinusoid curves (see Table 2) were significantly larger than 0 for children of 5 and 6 years old, both for Groups A and B (see Table 3; rejection of the H0 hypothesis), indicating that the amount of errors varied as a function of rotation angle and/ or direction of rotation. The H0 hypothesis was not rejected for the children at age 7 , so the $\mathrm{H} 1$ and $\mathrm{H} 2$ hypotheses were not tested at this age. However, at ages 5 and 6 for Group A and at age 5 for Group B, the phase shift parameters (see Table 2) were significantly larger than $90^{\circ}$ (reject the $\mathrm{H} 1$ hypothesis), but did not differ from $180^{\circ}$ (consistent with the $\mathrm{H} 2$ hypothesis; see Table 3). At these ages, response accuracy was thus shown to be affected by motor constraints, as evidenced by high numbers of errors for judging laterally rotated hands, compared to fewer mistakes for judging medially rotated hands (see Figure 2).

TABLE 2.

Fitted Parameters on the Erroneous Response Data for Group A and B

\begin{tabular}{ccccccc}
\hline & \multicolumn{3}{c}{ Group A } & \multicolumn{3}{c}{ Group B } \\
\cline { 2 - 7 } & Amplitude & $\begin{array}{c}\text { Phase } \\
\text { shift }\end{array}$ & Intercept & Amplitude & $\begin{array}{c}\text { Phase } \\
\text { shift }\end{array}$ & Intercept \\
\hline Age & 16.5 & 198 & 51.3 & 9.55 & 199 & 14.2 \\
5 & $(3.81)$ & $(13.2)$ & $(2.69)$ & $(3.59)$ & $(21.5)$ & $(2.54)$ \\
Age & 16.1 & 167 & 21.5 & 13.0 & 226 & 14.2 \\
6 & $(3.77)$ & $(13.4)$ & $(2.67)$ & $(4.52)$ & $(19.9)$ & $(3.20)$ \\
Age & 6.43 & 198 & 13.3 & 9.19 & 169 & 13.2 \\
7 & $(2.91)$ & $(26.0)$ & $(2.06)$ & $(4.55)$ & $(28.4)$ & $(3.22)$ \\
\hline
\end{tabular}

Note. Standard Error $(S E)$ in brackets. 


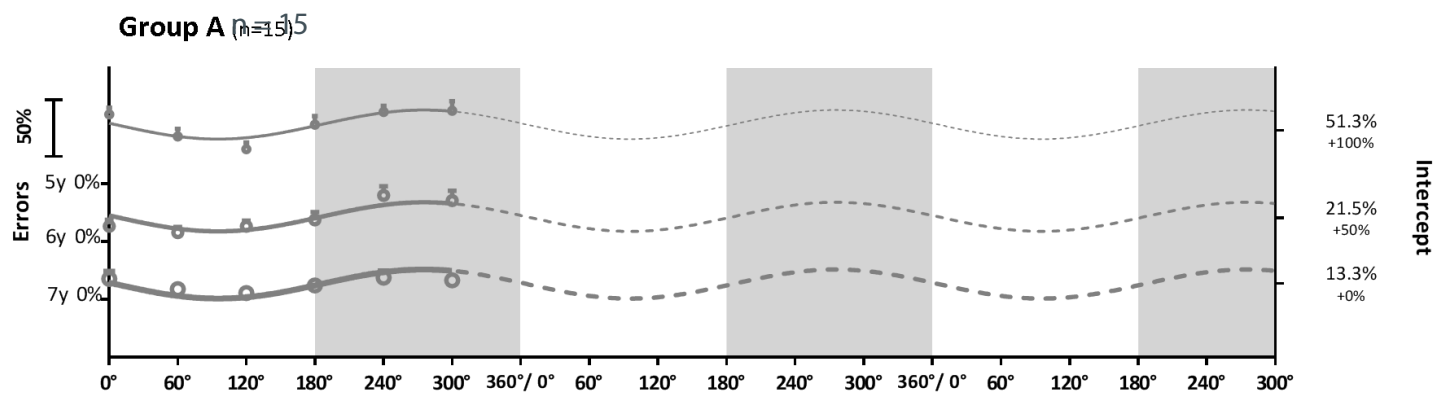

Group B $(A=8) 8$

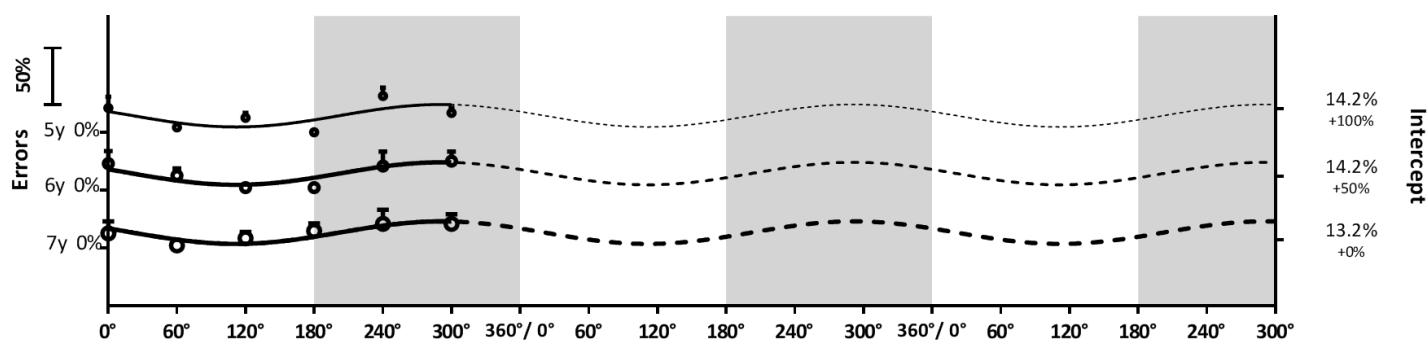

FIGURE 2.

Percentage of erroneous responses as a function of rotation angle. The solid lines represent the sinusoid curves through the observed error percentages. The dotted lines were added to depict the sinusoid nature of the a priori defined models. For better visualization, the curves are transposed as indicated on the right $y$ axis ( 5 years $+100 \% ; 6$ years $+50 \% ; 7$ years $+0 \%$ ). The data points present the mean percentage of errors and the $S E$ of the means per rotation angle. Grey areas represent laterally rotated stimuli.

TABLE 3.

F-tests of Goodness of Fit for the Fitted Parameters on the Percentage of Errors

\begin{tabular}{|c|c|c|c|c|c|c|c|}
\hline & \multirow{2}{*}{$\begin{array}{l}\text { Fitted parameter } \\
\text { tested against }\end{array}$} & \multicolumn{3}{|c|}{ Group A } & \multicolumn{3}{|c|}{ Group B } \\
\hline & & $\begin{array}{c}F \\
F \\
(1,87)\end{array}$ & $p$ & $\eta^{2}$ & $\begin{array}{c}F \\
(1,45)\end{array}$ & $p$ & $\eta^{2}$ \\
\hline \multirow{3}{*}{ Age 5} & Amplitude $\approx 0$ & 18.80 & $.0001^{*}$ & .402 & 7.09 & $.011^{*}$ & .202 \\
\hline & Phase shift $=90$ & 17.00 & $.0001^{*}$ & .378 & 6.31 & $.016^{*}$ & .184 \\
\hline & Phase shift $=180$ & 1.72 & .193 & .058 & .76 & .389 & .026 \\
\hline \multirow{3}{*}{ Age 6} & Amplitude $\approx 0$ & 18.20 & $.000^{*}$ & .394 & 8.30 & $.006^{*}$ & .229 \\
\hline & Phase shift $=90$ & 17.40 & $.000^{*}$ & .383 & 3.99 & .052 & .125 \\
\hline & Phase shift $=180$ & .87 & .353 & .030 & 4.31 & .044 & .134 \\
\hline \multirow{3}{*}{ Age 7} & Amplitude $\approx 0$ & 4.88 & .030 & .148 & 3.90 & .054 & .122 \\
\hline & Phase shift $=90$ & \# & \# & \# & \# & \# & \# \\
\hline & Phase shift $=180$ & \# & \# & \# & \# & \# & \# \\
\hline
\end{tabular}

Note. ${ }^{*}=$ significant $(p<.017$; Bonferroni corrected $)$; $=$ phase shift of 90 and phase shift of 180 were not tested when the amplitude did not differ from 0.

Therefore, motor imagery employment was indicated in these children, even when making incorrect judgments.

To examine developmental changes in employed strategies, we tested whether the parameters of the sinusoid curves describing the data changed across age. The amplitude $(p=.07)$ and phase shift $(p=$ .21) parameters did not change across age, indicating that the employed strategy remained similar between 5 and 7 years of age. However, it was found that the intercept parameters of Group A did change across age, $F(2,261)=64.39, p<.0001, \eta^{2}=.404$. This exemplifies a decrease in the average percentage of errors across age (i.e., from $51.3 \%$ at age 5 to $13.3 \%$ at age 7 , see Table 2). For Group B, none of the parameters changed between ages 5, 6, and 7 (amplitude $p=.77$; phase shift $p=.26$; intercept $p=.96$ ) indicating that not only the employed strategies were constant over age, but also the amount of errors (see Table 2).

\section{Response Durations}

For each age separately, response duration data of Groups A and B were fitted to a priori defined models that describe response duration pat- 
terns for different strategies. Table 4 presents the resulting fit equations and Figure 3 presents the complementary curves that were fitted on the response duration data for Groups A and B.

With the exception of the children in Group A at 5 years of age, the amplitude parameters (see Table 4 ) were significantly larger than 0 in both groups at all ages (see Table 4; rejection of the $\mathrm{H} 0$ hypothesis). Hence, except for children of Group A at 5 years of age, when they all performed inaccurately, response durations varied as a function of rotation angle and/or direction of rotation (see Figure 3). The phase shift parameters (see Table 4) were significantly larger than $90^{\circ}$ (rejection of the $\mathrm{H} 1$ hypothesis), but they did not significantly differ from $180^{\circ}$ (consistent with the $\mathrm{H} 2$ hypothesis; see Table 5). Figure 3 illustrates this effect of direction of rotation, as the response durations for judging

\section{TABLE 4.}

Fitted Parameters on the Response Durations of Group A and B

\begin{tabular}{ccccccc}
\hline & \multicolumn{3}{c}{ Group A } & \multicolumn{3}{c}{ Group B } \\
\cline { 2 - 7 } & Amplitude & $\begin{array}{c}\text { Phase } \\
\text { shift }\end{array}$ & Intercept & Amplitude & $\begin{array}{c}\text { Phase } \\
\text { shift }\end{array}$ & Intercept \\
\hline Age & 199 & 118 & 3,070 & 790 & 174 & 3,820 \\
5 & $(179)$ & $(51.6)$ & $(127)$ & $(285)$ & $(20.7)$ & $(201)$ \\
Age & 753 & 178 & 3,213 & 960 & 188 & 2,884 \\
6 & $(165)$ & $(12.6)$ & $(117)$ & $(192)$ & $(11.5)$ & $(136)$ \\
Age & 475 & 185 & 2,990 & 605 & 181 & 2,222 \\
7 & $(138)$ & $(16.6)$ & $(97.6)$ & $(92.4)$ & $(8.76)$ & $(65.3)$ \\
\hline
\end{tabular}

Note. Standard Error $(S E)$ in brackets. hands reached a maximum for laterally rotated hands (rotation angle of approximately $270^{\circ}$ ) and the durations are minimum for medially rotated hands (rotation angle of approximately $90^{\circ}$ ). These results thus provide indications for the use of motor imagery.

The amplitude parameters of Group A changed across age, $F(2$, $261)=13.83, p<.0001, \eta^{2}=.429$. However, the phase-shift parameters did not change across age $(p=.97)$. Put differently, direction of rotation predominantly affected the response durations in all three age groups, but the degree to which the direction of rotation affected the response duration differed across age. The intercepts also changed with age, $F(2$, $261)=82.79, p<.0001, \eta^{2}=.422$. Noteworthy, the response durations did not display an ongoing decrease as a function of age, as durations were shorter at age 5 than at age 6 (see Table 4 and Figure 3). For Group $\mathrm{B}$, the amplitude $(p=.48)$ and phase shift $(p=.77)$ parameters did not change between ages 5,6 , and 7 , indicating that the employed strategies were similar at these ages. The intercept changed with age, $F(2,135)$ $=30.55, p<.0001, \eta^{2}=.426$, indicating that the children did become faster from 5 to 7 years of age (see Table 4 and Figure 3 ).

\section{DISCUSSION}

In the present study, we examined the development of motor imagery strategies in the HLJ task in children in a longitudinal design by testing them once a year for three consecutive years at the ages of 5, 6, and 7 years. Children that performed the HLJ task at chance have conventionally been excluded from further analyses in previous studies (Butson
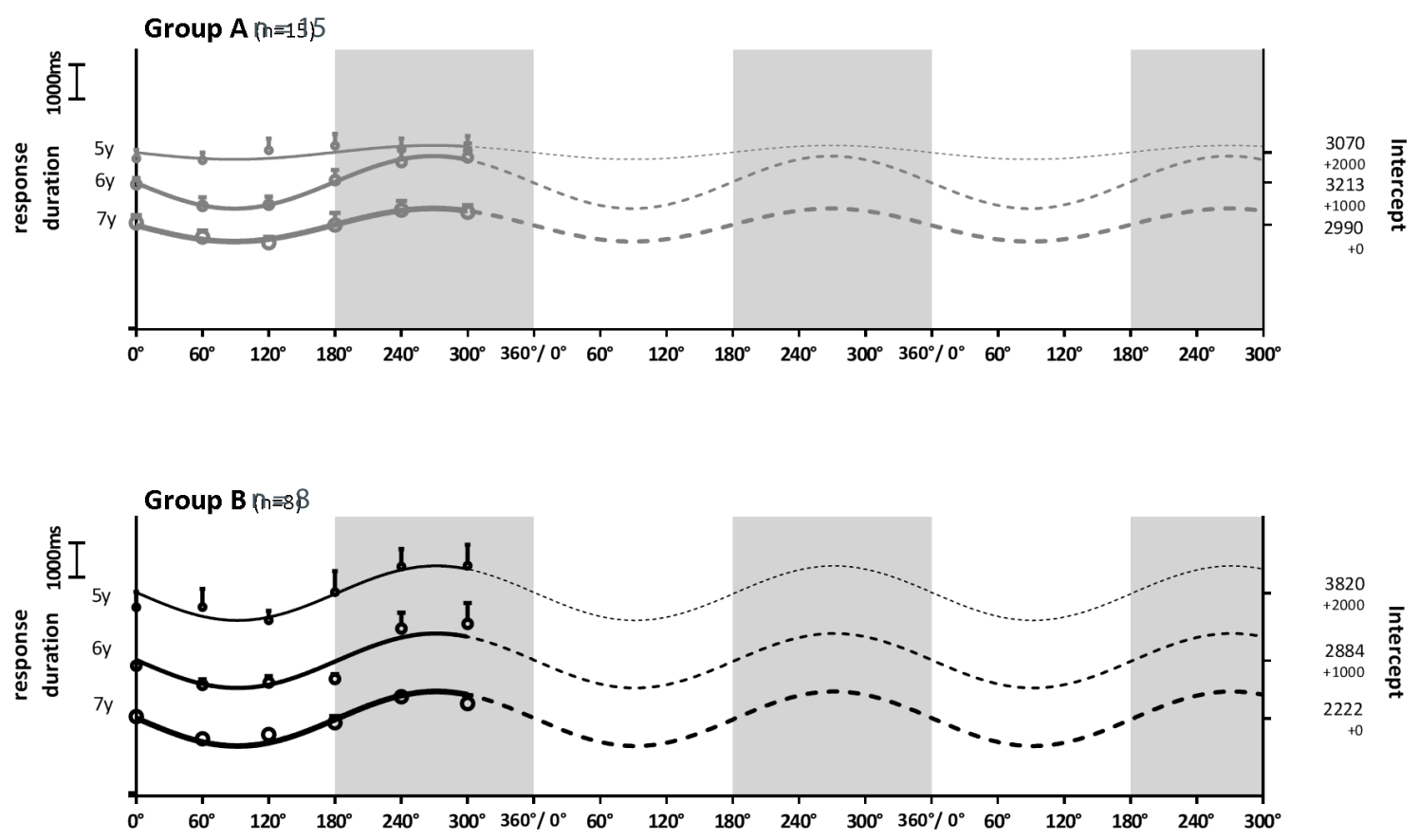

| FIGURE 3.

Response duration as a function of rotation angle. The solid lines represent the sinusoid curves through the observed response durations. The dotted lines were added to depict the sinusoid nature of the a priori defined models. For better visualization, the curves are transposed as indicated on the right $y$ axis ( 5 years $+2,000 ; 6$ years $+1,000 ; 7$ years +0 ). The data points present the mean percentage of errors and the $S E$ of the means per rotation angle. Grey areas represent laterally rotated stimuli. 


\section{TABLE 5.}

F-tests of Goodness of Fit for the Fitted Parameters on the Response Durations

\begin{tabular}{|c|c|c|c|c|c|c|c|}
\hline & \multirow{2}{*}{$\begin{array}{c}\text { Fitted parameter } \\
\text { tested against }\end{array}$} & \multicolumn{3}{|c|}{ Group A } & \multicolumn{3}{|c|}{ Group B } \\
\hline & & $\begin{array}{c}F \\
(1,87)\end{array}$ & $p$ & $\eta^{2}$ & $\begin{array}{c}F \\
(1,45)\end{array}$ & $p$ & $\eta^{2}$ \\
\hline \multirow{3}{*}{ Age 5} & Amplitude $\approx 0$ & 1.22 & .273 & .043 & 7.67 & $.008^{*}$ & .354 \\
\hline & Phase shift $=90$ & $\#$ & $\#$ & $\#$ & 7.62 & $.008^{\star}$ & .353 \\
\hline & Phase shift $=180$ & $\#$ & $\#$ & $\#$ & .07 & .787 & .005 \\
\hline \multirow{3}{*}{ Age 6} & Amplitude $\approx 0$ & 20.80 & $.0001^{*}$ & .426 & 25.00 & $.0001^{*}$ & .641 \\
\hline & Phase shift $=90$ & 20.80 & $.0001^{*}$ & .426 & 24.40 & $.0001^{*}$ & .636 \\
\hline & Phase shift $=180$ & .02 & .887 & .007 & .53 & .472 & .036 \\
\hline \multirow{3}{*}{ Age 7} & Amplitude $\approx 0$ & 11.90 & $.001^{*}$ & .298 & 43.00 & $.0001^{*}$ & .754 \\
\hline & Phase shift $=90$ & 11.80 & $.001^{*}$ & .296 & 42.80 & $.0001^{*}$ & .754 \\
\hline & Phase shift $=180$ & .08 & .783 & .003 & .01 & .950 & .0003 \\
\hline
\end{tabular}

Note. ${ }^{*}=$ significant $(p<.017$; Bonferroni corrected); $\#=$ phase shift of 90 and phase shift of 180 were not tested when the amplitude did not differ from 0.

et al., 2014; Funk, Brugger, \& Wilkening, 2005; Krüger \& Krist, 2009; Spruijt, Jongsma, et al., 2015). However, exclusion of children who do not judge hand laterality above chance might obscure a deeper insight into early developmental changes in imagery strategies. Therefore, we included children that performed at chance on the HLJ task at 5 years of age and examined the developmental changes in their performance, in addition to examining the children that did perform above chance at age 5. Since previous cross-sectional studies have not provided an unequivocal description of employed motor imagery strategies across age, we aimed at examining developmental changes in children's motor imagery strategies by using a longitudinal design over a period of three years. In what follows, we first discuss if overall HLJ task performance improved with age in terms of accuracy and response speed in both groups. Second, we discuss whether the error patterns, and whether the response duration patterns were random or whether they were according to a priori defined sinusoid models that reflect imagery strategies (see also Spruijt, Jongsma, et al., 2015). This was examined both for the children performing at and above chance at age 5. Finally, we discuss whether developmental changes in employed strategies can explain age-related improvements on the HLJ task performance.

The overall HLJ task accuracy improved across age in children from Group A but not Group B due to a floor effect with respect to the error percentage is the latter group. However, children in Group B, but not Group A, showed faster response times with age. These complementary findings suggest that there was a difference in the response-accuracy trade-off between the two groups. The children that were already able to accurately judge hand laterality at age 5 (Group B) became faster at judging hand laterality across age, as evidenced by developmental changes in the intercept of the sinusoid curves for the response durations (see also, e.g., Caeyenberghs, Tsoupas, et al., 2009; Spruijt, Jongsma, et al., 2015). Task accuracy, however, did not change across three consecutive years, as was illustrated by the consistently low percentage of errors at age 5, 6, and 7 (approximately $14 \%$, see Table 3 ). In contrast, children who did not perform the task accurately at age
5 (Group A) showed significant improvements on task accuracy and response speed between 5 and 7 years of age. Most of these children underwent a transition from not performing the task above chance at age 5 to performing the task above chance at the ages of 6 and 7 .

We first discuss developmental changes in employed imagery strategies for the children that were already accurate at age 5 , that is, above chance level (only one third of the children; Group B). It is important to note that for studying children who perform above chance, because of their low number of errors, response accuracy data reached a floor effect and were less meaningful in this group than the response speed data. Therefore, we focus on the response duration results in these children. The pattern of response durations was affected by motor constraints at age 5,6, and 7, indicating that children employed motor imagery. Importantly, the amplitude and phase shift did not differ across age. In line with our previous cross-sectional study, these longitudinal results thus confirm that the employed motor imagery strategy does not show developmental changes for children that accurately perform the HLJ task between 5 and 7 years of age (Spruijt, Jongsma, et al., 2015). These findings, however, diverge from previous studies that have shown age-related increases in motor imagery capability in children between 5 and 8 years of age (Krüger \& Krist, 2009; Toussaint et al., 2013). Apart from the employed design (cross-sectional vs. longitudinal), variations in used stimulus sets might have contributed to different findings (among these are back and/or palm view stimuli and different angles of rotation). In the current study, we used palm view hand pictures that are more difficult to judge compared to back view hand pictures (Ter Horst et al., 2010). In addition, we found that judging back view hand pictures relies predominantly on visual recognition instead of a motor imagery strategy (Ter Horst et al., 2010). Thus, we used palm view stimuli only in the current study, as we were interested in the development of motor imagery capacity. A likely reason for the differences in study results is the analysis methods for determining the employed strategies based on the response duration and response accuracy data. Whereas most previous studies separately considered the 
effect of rotation angle and direction of rotations (e.g., Funk et al., 2005; Krüger \& Krist, 2009; Toussaint et al., 2013), the current approach considers the cumulative effects of these factors (see also Spruijt, Jongsma, et al., 2015).

Previous HLJ task studies excluded children who performed at chance from further analyses, without discussing the underlying reason for doing so (Butson et al., 2014; Funk et al., 2005; Krüger \& Krist, 2009; Spruijt, Jongsma, et al., 2015). The exclusion of these children suggests that researchers (perhaps implicitly) interpreted this as a lack of ability to use motor imagery. As a critical extension of these studies, we also examined the employed motor imagery strategies for children that did not perform above chance at 5 years of age. Before considering developmental changes in imagery strategies in these children, we first address the question whether inaccurate performance on the HLJ task is indeed caused by an inability to employ motor imagery (see Deconinck, Spitaels, Fias, \& Lenoir, 2009; Williams, Thomas, Maruff, \& Wilson, 2008). Alternatively, an inability to understand task instructions or inaccuracy while employing motor imagery can underlie inaccurate HLJ task performance. In line with Butson et al. (2014), the majority of 5 -year-old participants did not perform above chance. It is important to note that for studying the employed strategies in children who did not perform above chance, the error data might be more reliable than response duration data. That is, in line with the speed-accuracy tradeoff, the 5-year-olds who responded at chance (high numbers of errors; Group A) responded relatively fast in comparison with their peers who performed accurately (Group B; see Table 4 and Figure 3). Because of these fast responses and concomitant low accuracy, we propose that the error data are more representative of the employed imagery strategies compared to the response duration data. It was found that task accuracy, that is, the error pattern, was clearly affected by motor constraints in children performing at chance at age 5. Hand stimuli representing biomechanically less awkward (medial) rotations more often led to correct responses than those with more awkward (lateral) rotations, indicating the use of motor imagery (see also Ter Horst et al., 2010). We can therefore reject the hypothesis that the children did not understand the HLJ task, which would have resulted in blind guesses (i.e., no structure in the pattern of errors) to perform the task (see also Mutsaarts, Steenbergen, \& Bekkering, 2007). It can thus be concluded that even though children perform the HLJ task inaccurately at young age, they already have mental representations of hand movements and are able to access them for judging hand laterality. Hence, involvement of motor imagery is not the rate limiter (the slowest developing factor that affects how well an individual can exhibit a motor behaviour, see Thelen \& Smith, 1994) for accurately performing HLJs. Alternatively, more general cognitive abilities might hinder HLJ task performance (see Spruijt, Jongsma, et al., 2015). For instance, attention might be a rate limiter for the HLJ task at young age because judging hand laterality through the internal activation of mental representations of hand movements has been suggested to place large demands on children's attention (Schott, 2012).

Determining developmental changes in employed strategies is particularly of interest in children of Group A, as most of these children underwent a transition from not performing the task above chance at age 5 towards performing the task above chance at age 6 and 7. Yet, we did not observe developmental changes in the strategy that the children employed at age 5,6, and 7. Above, we already discussed that response accuracy patterns indicated motor imagery involvement at age 5. At age 6 and 7 (most children then performed above chance), response durations were largest for stimuli in biomechanically awkward (lateral) rotations, indicating the use of motor imagery (see also Ter Horst et al., 2010). Furthermore, we found that the phase shifts for the sinusoid curves of Group A did not vary as a function of age, neither for the error data nor for the response duration data. Hence, no developmental changes in the employed motor imagery strategies were observed between 5 and 7 years of age. Consequently, the observed improvements in overall performance on the HLJ task (faster and more accurate responses) in young children cannot be attributed to developmental changes in the employed motor imagery strategy to perform the HLJ task. Instead, we argue that HLJ task improvements might be attributed to the development of cognitive abilities that can influence HLJ task performance during childhood. As was already discussed, the process of mentally representing hand movements in order to judge hand laterality places large demands on children's attention (Schott, 2012). HLJ task improvements between 5 and 7 years of age might therefore be linked to improvements in attention processes across age (Breckenridge, Braddick, \& Atkinson, 2013; Levy, 1980). In a similar fashion, as motor imagery involves activation of movement representations in working memory (Decety \& Grezes, 1999; Munzert, Lorey, \& Zentgraf, 2009), working memory capacity might affect HLJs (see also Gabbard, Lee, \& Cacola, 2013; Schott, 2012). Since working memory is developing during childhood (Kemps, De Rammelaere, \& Desmet, 2000), these developmental changes might underlie the improvements on the HLJ task between 5 and 7 years of age.

It is important to point out that the HLJ task is not the only paradigm to study motor imagery. In fact, in our recent paper (Spruijt, Jongsma, et al., 2015) we have discussed the pros and cons of the different paradigms that are used to study motor imagery. Still, we used the HLJ task for two obvious reasons. First, it is the most commonly used paradigm in children allowing comparison among studies and, second, it is an implicit way to determine motor imagery, which is very suitable for children.

To conclude, children's HLJ task performance is affected by motor constraints at age 5. Motor representations that are involved in the planning and feedforward control of movement (Jeannerod, 1994; Vogt, Rienzo, Collet, Collins, \& Guillot, 2013; Wolpert, 1997) are thus formed and can already be accessed at 5 years of age. This accords well with previous indications that the majority of 5-year-old children are able to plan their movements (Weigelt \& Schack, 2010), use feedforward control (De Ste Croix \& Korff, 2012), and the dual-action simulation account of imagery during observation (Eaves, Riach, Holmes, \& Wright, 2016).

In order to examine the early development of motor imagery, we extended previous studies by additionally examining the large proportion of children that did not perform above chance level at the HLJ task 
at age 5 . We observed that children were engaged in motor imagery to perform the HLJ task, even when this led to a high proportion of erroneous responses. We thereby demonstrate that motor imagery ability is not the limiting factor for accurate HLJ task performance. Furthermore, the use of motor imagery to judge hand laterality did not change between ages 5 and 7 years, neither for the children who performed consistently accurately (in accordance with our previous cross-sectional findings) nor for the children who were not accurate at age 5. Consequently, it can be concluded that once children are able to activate movement representations, the use of this motor imagery strategy for performing the HLJ task does not change across age. The improvements in accuracy and speed on the HJL task across age can therefore not be attributed to developmental changes in the use of motor imagery. Alternatively, the development of more general cognitive processes like working memory and attention might underlie the development of children's HLJ task performance. Future studies should employ other paradigm tasks in order to evaluate whether the current findings generalize to other measures of motor imagery.

These findings have implications for (clinical) practice as well. A feature of the present study is the individual variation among children to enlist motor imagery. Future work, building on the method that is presented here, may help to identify children that are able to use motor imagery, and are therefore eligible for interventions based on motor imagery (Wilson et al., 2016). These interventions have shown to be effective and become increasingly used in (clinical) practice.

\section{REFERENCES}

Breckenridge, K., Braddick, O., \& Atkinson, J. (2013). The organization of attention in typical development: A new preschool attention test battery. British Journal of Developmental Psychology, 31, 271-288. doi: 10.1111/bjdp.12004 |wWW

Butson, M. L., Hyde, C., Steenbergen, B., \& Williams, J. (2014). Assessing motor imagery using the hand rotation task: Does performance change across childhood? Human Movement Science, 35, 50-65. doi: 10.1016/j.humov.2014.03.013 |WWW

Caeyenberghs, K., Tsoupas, J., Wilson, P. H., \& Smits-Engelsman, B. C. (2009). Motor imagery development in primary school children. Developmental Neuropsychology, 34, 103-121. doi: 10.1080/87565640802499183 $\overline{\text { WWW }}$

Caeyenberghs, K., Wilson, P. H., van Roon, D., Swinnen, S. P., \& Smits-Engelsman, B. C. (2009). Increasing convergence between imagined and executed movement across development: Evidence for the emergence of movement representations. Developmental Science, 12, 474-483. doi: 10.1111/j.14677687.2008.00803.x

Decety, J., \& Grezes, J. (1999). Neural mechanisms subserving the perception of human actions. Trends in Cognitive Sciences, 3, 172-178. doi:S1364-6613(99)01312-1 $\underline{\underline{W W}}$

Deconinck, F. J., Spitaels, L., Fias, W., \& Lenoir, M. (2009). Is developmental coordination disorder a motor imagery deficit? Journal of Clinical and Experimental Neuropsychology, 31, 720-730. doi: $10.1080 / 13803390802484805$ WWW
De Ste Croix, M., \& Korff, T. (2012). Paediatric biomechanics and motor control-Theory and application. London, England: Routledge.

Eaves, D. L., Riach, M., Holmes, P. S., \& Wright, D. J. (2016). Motor imagery during action observation: A brief review of evidence, theory and future research opportunities. Frontiers in Neuroscience, 10:514. doi: 10.3389/fnins.2016.00514 WWW

Funk, M., Brugger, P., \& Wilkening, F. (2005). Motor processes in children's imagery: The case of mental rotation of hands. Developmental Science, 8, 402-408. doi: 10.1111/j.1467-7687 2005.00428.x $\overline{\mathrm{WWW}}$

Gabbard, C., Lee, J., \& Cacola, P. (2013). Role of working memory in transformation of visual and motor representations for use in mental simulation. Cognitive Neuroscience, 4, 210-216. doi: 10.1080/17588928.2013.820180 $\underline{\underline{W W} \mid}$

Geuze, R. H., Lust, J. M., \& Bouma, A. (2009). Een handvoorkeurstest voor kinderen van 5 tot 7 jaar [Hand preference test for 5 to 7 year old children]. Tijdschrift voor Neuropsychologie, 1, 43-51. Grammer, J. K., Coffman, J. L., Ornstein, P. A., \& Morrison, F. J. (2013). Change over time: Conducting longitudinal studies of children's cognitive development. Journal of Cognition and Development, 14, 515-528. doi: 10.1080/15248372.2013.833925 WWW

GraphPad Prism (Version 6.07) [Computer software]. La Jolla, CA: GraphPad Software.

Hétu, S., Grégoire, M., Saimpont, A., Coll, M-P., Eugène, F., Michon, P-E., \& Jackson, P. L. (2013). The neural network of motor imagery: An ALE meta-analysis. Neuroscience \& Biobehavioral Reviews, 37, 930-949. doi: 10.1016/j.neubiorev.2013.03.017 WWW

Jeannerod, M. (1994). The representing brain-Neural correlates of motor intention and imagery. Behavioural and Brain Sciences, 17, 187-202. doi: 10.1017/S0140525X00034026

Jeannerod, M. (1995). Mental imagery in the motor context. Neuropsychologia, 33, 1419-1432. doi:0028-3932(95)00073-C WWW

Jongsma, M. L. A., Meulenbroek, R. G. J., Okely, J., Baas, C. M., van der Lubbe, R. H. J., \& Steenbergen, B. (2013). Effects of hand orientation on motor imagery-Event related potentials suggest kinesthetic motor imagery to solve the hand laterality judgment task. PLOS ONE, 8:e76515. doi: 10.1371/journal. pone.0076515. WWW

Kemps, E., De Rammelaere, S., \& Desmet, T. (2000). The development of working memory: Exploring the complementarity of two models. Journal of Experimental Child Psychology, 77, 89-109. doi: 10.1006/jecp.2000.2589 WWW

Krüger, M., \& Krist, H. (2009). Imagery and motor processesWhen are they connected? The mental rotation of body parts in development. Journal of Cognition and Development, 10, 239-261. doi: 10.1080/15248370903389341

Levy, F. (1980). The development of sustained attention (vigilance) and inhibition in children: Some normative data. Journal of Child Psychology and Psychiatry, 21, 77-84. Retrieved from http://www.ncbi.nlm.nih.gov/pubmed/7358807 WWW 
Lust, J. M., Geuze, R. H., Wijers, A. A., \& Wilson, P. H. (2006). An EEG study of mental rotation-related negativity in children with Developmental Coordination Disorder. Child: Care, Health and Development, 32, 649-663. doi: 10.1111/j.1365-2214 .2006.00683.x WWW

Munzert, J., Lorey, B., \& Zentgraf, K. (2009). Cognitive motor processes: The role of motor imagery in the study of motor representations. Brain Research Reviews, 60, 306-326. doi: 10.1016/j. brainresrev.2008.12.024 WWW

Mutsaarts, M., Steenbergen, B., \& Bekkering, H. (2007). Impaired motor imagery in right hemiparetic cerebral palsy. Neuropsychologia, 45, 853-859. doi 10.1016/j. neuropsychologia.2006.08.020 [WWW

Oldfield, R. C. (1971). The assessment and analysis of handedness: The Edinburgh inventory. Neuropsychologia, 9, 97-113. doi: 10.1016/0028-3932(71)90067-4 WW

Parsons, L. M. (1987). Imagined spatial transformations of one's hands and feet. Cognitive Psychology, 19, 178-241. doi: 10.1016/0010-0285(87)90011-9 wW

Schott, N. (2012). Age-related differences in motor imagery: Working memory as a mediator. Experimental Aging Research, 38, 559-583. doi: 10.1080/0361073X.2012.726045 |WWW

Shepard, R. N., \& Metzler, J. (1971, February). Mental rotation of three-dimensional objects. Science, 171(3972), 701-703. doi: 10.1126/science.171.3972.701 $\overline{\text { WWW }}$

Smits-Engelsman, B. C., \& Wilson, P. H. (2012). Age-related changes in motor imagery from early childhood to adulthood: Probing the internal representation of speed-accuracy tradeoffs. Human Movement Science, 32, 1151-1162. doi: 10.1016/j. humov.2012.06.006 $\overline{\mathrm{WWW}}$

Spruijt, S., Jongsma, M. L., van der Kamp, J., \& Steenbergen, B. (2015). Predictive models to determine imagery strategies employed by children to judge hand laterality. PLOS ONE, 10:e0126568. doi: 10.1371/journal.pone.0126568 WWW

Spruijt, S., Jouen, F., Molina, M., Kudlinski, C., Guilbert, J., \& Steenbergen, B. (2013). Assessment of motor imagery in cerebral palsy via mental chronometry: The case of walking. Research in Developmental Disabilities, 34, 4154-4160. doi: 10.1016/j.ridd.2013.08.04 $\overline{\mathrm{WWW}}$

Spruijt, S., van der Kamp, J., \& Steenbergen, B. (2015). Current insights in the development of children's motor imagery ability. Frontiers in Psychology, 6:787. doi: 10.3389/fpsyg.2015.00787 WWW

Steenbergen, B., van Nimwegen, M., \& Crajé, C. (2007). Solving a mental rotation task in congenital hemiparesis: Motor imagery versus visual imagery. Neuropsychologia, 45, 3324-3328. doi: 10.1016/j.neuropsychologia.2007.07.002 WWW

Ter Horst, A. C., Jongsma, M. L. A., Janssen, L. K., van Lier, R., \& Steenbergen, B. (2012). Different mental rotation strategies reflected in the rotation related negativity. Psychophysiology, 49, 566-573. doi: 10.1111/j.1469-8986.2011.01322.x WWW

Ter Horst, A. C., van Lier, R., \& Steenbergen, B. (2010). Mental rotation task of hands: Differential influence number of rotational axes. Experimental Brain Research, 203, 347-354. doi: 10.1007/

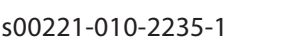

Ter Horst, A. C., van Lier, R., \& Steenbergen, B. (2013). Mental rotation strategies reflected in event-related (de) synchronization of alpha and mu power. Psychophysiology, 50, 858-863. doi:

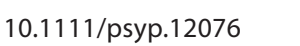

Thelen, E., \& Smith, L. B. (1994). A dynamic systems approach to the development of cognition and action. Cambridge, MA: MIT Press.

Toussaint, L., Tahej, P. K., Thibaut, J. P., Possamai, C. A., \& Badets, A. (2013). On the link between action planning and motor imagery: A developmental study. Experimental Brain Research, 231, 331-339. doi: 10.1007/s00221-013-3698-7 WWW

Vogt, S., Rienzo, F. D., Collet, C., Collins, A., \& Guillot, A. (2013). Multiple roles of motor imagery during action observation. Frontiers in Human Neuroscience, 7:807. doi: 10.3389/ fnhum.2013.00807 WWW

Weigelt, M., \& Schack, T. (2010). The development of endstate comfort planning in preschool children. Experimental Psychology, 57, 476-482. doi: 10.1027/1618-3169/a000059 WWW

Williams, J., Thomas, P. R., Maruff, P., \& Wilson, P. H. (2008). The link between motor impairment level and motor imagery ability in children with developmental coordination disorder. Human Movement Science, 27, 270-285. doi: 10.1016/j. humov.2008.02.008 WWW

Wilson, P. H., Adams, I. L. J., Caeyenberghs, K., Williams, J., Thomas, P., Smits-Engelsman, B., \& Steenbergen, B. (2016). Motor imagery training enhances motor skill in children with DCD: $A$ replication study. Research in Developmental Disabilities, 57,

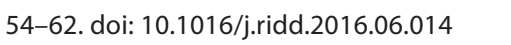

Wilson, P. H., Maruff, P., Butson, M., Williams, J., Lum, J., \& Thomas, P. R. (2004). Internal representation of movement in children with developmental coordination disorder: A mental rotation task. Developmental Medicine and Child Neurology, 46, 754-759. Retrieved from http://www.ncbi.nlm.nih.gov/

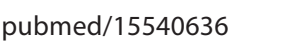

Wohlwill, J. F. (1970). Methodology and research strategy in the study of developmental change. In L. R. Goulet \& P. B. Baltes (Eds.), Life-span developmental psychology: Research and theory (pp. 149-191). New York, NY: Academic Press.

Wolpert, D. M. (1997). Computational approaches to motor control. Trends in Cognitive Sciences, 1, 209-216. doi: 10.1016/ S1364-6613(97)01070-X $\underline{\underline{W W}}$

RECEIVED 30.11.2016 | ACCEPTED 22.08.2017 